Mots. Les langages du politique

\title{
Brandir un crayon, geste-emblème des rassemblements post-attentats de janvier 2015
}

Brandishing a pencil, emblem-gesture of the rallies after the January 2015 attacks

Esgrimir un lápiz, un gesto emblemático de las concentraciones post-atentados de enero del 2015

\section{Maëlle Bazin}

\section{OpenEdition}

\section{Journals}

Édition électronique

URL : https://journals.openedition.org/mots/22227

DOI : $10.4000 /$ mots. 22227

ISSN : 1960-6001

Éditeur

ENS Éditions

\section{Édition imprimée}

Date de publication : 9 mai 2016

Pagination : 67-82

ISBN : 978-2-84788-793-8

ISSN : 0243-6450

\section{Référence électronique}

Maëlle Bazin, « Brandir un crayon, geste-emblème des rassemblements post-attentats de

janvier 2015 », Mots. Les langages du politique [En ligne], 110 | 2016, mis en ligne le 09 mai 2018, consulté le 23 avril 2022. URL : http://journals.openedition.org/mots/22227 ; DOI : https://doi.org/ $10.4000 /$ mots. 22227 


\section{Brandir un crayon, geste-emblème des rassemblements post-attentats de janvier 2015}

Mercredi 7 janvier 2015, le soir des attentats à Charlie Hebdo, des centaines de personnes se réunissent dans les villes et villages de France. À Paris, la place de la République est noire de monde. Au sein de cette foule endeuillée, nombreux sont ceux qui, le bras levé au-dessus de la tête, brandissent un crayon. Ce geste simple relève, dans sa forme collective, d'un mouvement de deuil et de protestation à la suite de la série d'attaques terroristes islamistes intervenues dans la capitale française. Cet article interroge la circulation, les pratiques et les représentations d'une gestuelle de solidarité qui condense à la fois un hommage aux victimes, une défense de la liberté d'expression et une lutte contre le terrorisme.

Si les mobilisations collectives qui ont suivi les attentats ont été désignées sous les termes de marches - notamment pour celles du dimanche 11 janvier -, les journalistes ont dans le même temps identifié les participants à des manifestants ${ }^{1}$. Les marches en tant que démonstrations collectives s'appuient sur une scénographie manifestante composée d'un arsenal de signes : drapeaux, banderoles et autres supports de messages, slogans criés, chants, silences, mais également de gestes politiques dont le crayon brandi. Dans un chapitre intitulé «Quand faire c'est dire », Olivier Fillieule et Danielle Tartakowsky parlent, à propos de la manifestation, d' " un langage qui s'inscrit dans une dramaturgie », « une expression en acte d'une opinion politique» (2013, p.167). Les participants aux rassemblements post-attentats mobilisent ainsi plusieurs modes d'expression : l'écriture, la parole et des gestuelles particulières. Nous reprendrons l'hypothèse soulevée par Emmanuel Soutrenon selon laquelle «ce n'est pas tout à fait la même chose de manifester avec son corps qu'avec du papier (pamphlets, tracts, pétitions...), et que le médium choisi pour exprimer sa manifestation n'est pas sans effet sur celle-ci, sur sa structuration, voir sur les effets qu'elle produit» (1998, p. 38). Ce travail s'inscrit dans une approche

1. M. Pigenet et $D$. Tartakowsky relèvent un glissement sémantique entre «manifestation » et «marche» à partir des années quatre-vingt, où les termes sont utilisés de façon interchangeable (2003, p. 91).

CARISM (Centre d'analyse et de recherche interdisciplinaire sur les médias) bazinmael@gmail.com 
transdisciplinaire empruntant aux champs de la linguistique, de la sémiotique, de la sociologie des mouvements sociaux et de l'analyse de discours pour nourrir une réflexion sur une forme de réponse collective à un événement tragique dans l'espace public. Quels sont les enjeux à la fois symboliques et politiques de la gestuelle du crayon brandi ? Et comment cet ensemble geste-objet a-t-il été représenté, identifié, nommé, légitimé par les acteurs en présence?

Avant d'interroger la nature du geste et ses significations, nous partirons de l'objet brandi sans qui cette gestuelle de monstration n'aurait pas de raison d'être. Qu'ils soient de production industrielle ou artisanale, les crayons, outils de la contestation, symbolisent la liberté d'expression. Le crayon avait déjà été mis en scène comme défenseur des droits des journalistes par le biais des travaux de Cartonning for Peace, notamment. Avec la volonté de joindre le geste à l'objet, nous comparerons les pratiques effectives lors des marches et leurs diverses représentations. Arborer ou brandir : la manière de tenir le crayon influe sur sa signification. Nous interrogerons également l'apparente spontanéité du crayon brandi et ses enjeux, variables selon le contexte de réalisation. Nous mettrons enfin en relief le processus de désignation et de légitimation de cette gestuelle à travers une analyse de la photographie de Stéphane Mahé, Le crayon guidant le peuple, en tant qu'image ancrée dans un imaginaire collectif et présentant une double référentialité, indicielle et iconique.

\section{Le crayon : outil de la contestation}

\section{Du crayon manufacturé au crayon augmenté}

Crayons et stylos, ces deux outils d'expression se sont côtoyés dans les rassemblements post-attentats. Malgré leur diversité de formes et d'usages, l'un plutôt pour le dessin, l'autre plutôt pour l'écriture, on constate la prédominance du crayon (du dessinateur) dans les représentations des événements et dans les commentaires qui y sont associés.

À la maison, à l'école, au travail, le crayon fait partie de notre vie quotidienne. Au sein des mobilisations de janvier, on peut en identifier trois types, non pas employés dans leur fonction utilitaire mais symbolique. Premièrement, les crayons usuels, de ceux que l'on glisse facilement dans une poche, un sac ou un agenda. Crayons que les manifestants portaient probablement déjà sur eux en venant à la marche (élément qui peut expliquer le succès de ce geste), et qui, une fois l'émotion retombée, ont repris leur place initiale ou ont été déposés au pied de mémoriaux improvisés ${ }^{2}$ sans que leurs propriétaires puissent

2. Les archives municipales de Rennes ont récolté plusieurs centaines d'outils d'écriture en tout genre (crayons à papier, stylos à bille, marqueurs, effaceurs, feutres, pinceaux, craies) lors de la collecte des messages et objets déposés place de la mairie. 
se remémorer lequel du «Bic » ou du crayon à papier a été brandi. Puis il y a les crayons estampillés «Charlie», spécifiquement créés dans l'urgence par des industriels. Divers sites en ligne en proposent à la vente et reversent - parfois, pas toujours - une partie des recettes à Charlie Hebdo ou à des associations de défense des journalistes, notamment l'un d'eux qui offre pour moins de deux euros la perspective «de changer le monde». À la fois action de solidarité et objet de souvenir, ils reprennent les codes graphiques du slogan «Je suis Charlie », baguette de bois noire et écriture blanche. Ces objets s'inscrivent dans le régime des goodies, autrement dit du gadget publicitaire siglé au nom de l'entreprise. Charlie devient une marque. Enfin, la troisième catégorie ne relève pas d'une production industrielle mais artisanale. Certains manifestants ont bricolé en carton ou dans d'autres matériaux rudimentaires des crayons géants, des versions augmentées faites maison. De taille variable, allant de quelques centimètres de diamètre, facilement préhensibles, à plusieurs mètres, comme celui acheminé par la foule place de la Nation - visible notamment sur la photographie de Martin Argyroglo3 - ces objets n'ont aucune fonction d'écriture, seule la portée symbolique subsiste. Faits de tubes, de manches à balai et d'objets de récupération divers, ils sont souvent peints de slogans comme «liberté » ou «Charlie». Ces objets-instruments relèvent de ce que Gavin Grindon désigne sous la formule «objets désobéissants». En 2014, il a travaillé avec Catherine Flood pour le Victoria and Albert Museum de Londres sur la première exposition traitant du rôle des objets dans les mouvements contestataires mondiaux 4 . Les objets désobéissants existent depuis que les luttes sociales existent. Ils sont des moyens, pour les protestataires, de se faire entendre. Outils de la contestation, ils démontrent un investissement particulier des participants. "Ils sont fonctionnels, créatifs et s'inscrivent dans l'ère de la consommation. C'est un design populaire assez brut, avec des objets réalisés sur le moment, dans l'action, par des non-professionnels, avec les moyens du bord $» 5$.

Outre ces objets produits par les manifestants, certaines communes introduisent des éléments matériels symboliques dans l'espace public, prolongeant l'élan des marches républicaines. Les initiatives abondent, comme pour la ville vosgienne d’Épinal qui a taillé le sapin de Noël de la place principale en forme de crayon. Intervention de la tronçonneuse qui opère un glissement symbolique d'une décoration de tradition chrétienne à la liberté d'expression. Graffitée par les passants, photographiée et multidiffusée sur le Web, cette représentation, haute de cinq mètres, de l'outil des dessinateurs, vient enrichir

3. «Nation », janvier 2015, M. Argyroglo (http://www.martin-argyroglo.com/198129/7239950/ perso/marche-du-11-janvier).

4. Exposition Disobedient Objects, Victoria and Albert Museum, Londres, 26 juillet 2014 au 1 er février 2015 .

5. C. Maliszewski, 2014, «Gavin Gindron : “L’objet désobéissant aide les protestataires à se faire entendre" ", lemonde.fr. 
l'iconographie prolifique du crayon comme symbole de la «libre communication des pensées et des opinions ${ }^{6}$.

\section{Symbole de la liberté d'expression}

Contrairement à la marche des crayons rouges de janvier 2014, au cours de laquelle deux cents journalistes et activistes malaisiens ont brandi des crayons cassés en deux pour symboliser la violence du gouvernement contre les médias7, lors des rassemblements post-attentats, les crayons sont bel et bien entiers. Il s'agit non pas de dénoncer un système de censure mais de défendre une profession et une valeur républicaine tout en rendant hommage aux dessinateurs assassinés. De nombreux dessins de presse ont illustré l'attentat contre les membres de Charlie Hebdo par un crayon brisé, mais ce, pour mieux rebondir sur l'idée d'une continuité de l'activité (journalistique) et de la lutte (pour la liberté d'expression) : le crayon surpassant sa cassure est alors représenté démultiplié ou poursuivant son tracé8. Le crayon comme outil du dessinateur et le stylo comme instrument du journaliste9 représentent les moyens d'une expression libre, condition nécessaire à l'établissement et au fonctionnement de la démocratie. Certains journalistes ont même brandi leur carte de presse pour souligner leur présence et afficher leur détermination. «C'est avec un crayon qu'on dessine, mais aussi qu'on écrit des lettres, des mots, et en particulier notre langue française avec laquelle tant d'écrivains et d'intellectuels ont formulé des idées et des textes ", relève Jérôme Clément, président de la fondation Alliance française ${ }^{10}$. La figure de l'intellectuel est valorisée comme contributeur actif à l'épanouissement de la société. De Voltaire à Alfred Jarry, en passant par Charles Baudelaire, plusieurs grands auteurs sont d'ailleurs cités dans les rassemblements. Ces messages font l'éloge de la culture française et des personnes ayant œuvré en sa faveur par leur plume. «[Liberté], j’écris ton nom », le refrain du poème de Paul Éluard, recopié sur les pancartes et graffité sur les murs des villes, s'inscrit alors comme vers performatif.

Mais le crayon, c'est aussi le rappel d'une pratique enfantine commune à tous, car «avant même de parler correctement et longtemps avant de savoir écrire, tous, nous avons eu un crayon en main et gribouillé des dessins»

6. Déclaration universelle des Droits de l'homme de 1948.

7. Cette manifestation fait suite à une décision ministérielle de suspendre le magazine d’information The Heat en représailles, selon les protestataires, contre la publication d'un article sur les habitudes de dépenses du Premier ministre et de sa femme.

8. Voir, entre autres, le dessin de Lucille Clerc que plusieurs journalistes ont attribué par erreur à Banksy : yesterday représenté par un crayon entier, today par un crayon brisé et tomorow avec deux crayons issus de la cassure (http://lucilleclerc.com/lucilleclerc-07-01-15.html).

9. Si, dans la réalité des pratiques, les outils numériques ont complété voir supplanté les outils classiques d'écriture, le crayon et le stylo restent des représentations symboliques de ces métiers.

10. Allocution de Jérôme Clément lors du $36^{\mathrm{e}}$ colloque de la Fondation Alliance française le lundi 26 janvier 2015 . 
(Stibbe, 2015). Barbouillages, griffonnages, beaucoup de dessins d'enfants étaient affichés dans les mobilisations. Généralement cantonnées au mur de la classe ou fièrement aimantées sur la porte du réfrigérateur familial, ces expressions naïves trouvent une valorisation particulière dans les rassemblements; et ces contributions participent, dans un sens, à une perception positive du dessinateur de presse.

Enfin, le crayon est l'instrument de la signature par lequel les endeuillés viennent écrire leurs messages de condoléances dans les nombreux livres d'or laissés à disposition du public. Il condense d'autant plus la charge affective lorsqu'il devient un objet de deuil. On observe une accumulation de crayons et de stylos dans les lieux de recueillement éphémères comme la place de la République, le siège du journal satirique ou les parvis des mairies et Hôtels de Ville en province. Au milieu des amas de bougies allumées ou consumées, de fleurs, de dessins, de messages, ils jonchent le sol, tels des offrandes donnant à ces espaces un caractère d'autel (Doss, 2008). L'amoncellement crée ici une force particulière. On remarque aussi, avec ces mêmes crayons, la formation de mots («Liberté», «Charlie») ou de symboles (Peace and love) au pied des mémoriaux officieux. Forme d'écriture monumentale à travers laquelle les manifestants réalisent une mise en abyme de la liberté d'expression.

Décorant des pancartes, accrochés sur les vêtements ou dans les cheveux des participants, les crayons sont très présents dans les rassemblements de janvier. Tels des insignes, ils servent de signes distinctifs, commémorant un événement et représentant l'appartenance à un groupe. Si la symbolique est invariablement celle de la liberté d'expression, la valeur de l'acte diffère quand celui-ci est pris dans une rhétorique de monstration particulière : la main levée ou le poing levé.

\section{Joindre le geste à l'objet}

Contrairement aux marches du 11 janvier qui étaient annoncées et planifiées, lors des premiers rassemblements, le mercredi 7, jour de l'attentat à Charlie Hebdo, les participants disposaient de peu de signalétique, à savoir drapeaux, pancartes ou autres objets de fabrication personnelle ${ }^{11}$. Sans point de départ identifiable, la gestuelle du crayon brandi semble émaner du collectif. Elle relève d'une forme de spontanéité par la rapidité de la réponse donnée à un événement soudain, inattendu et tragique. Ce caractère impulsif a été maintes fois souligné par les médias. Orpheline, cette gestuelle apparaît comme d'autant plus légitime. Elle serait une forme de démonstration physique d'une France unie, entrant en communion par l'adoption de comportements

11. À noter que le slogan «Je suis Charlie » était déjà présent. 
similaires. Mais bien que non soumise à prescription ou encadrement par une autorité ou une organisation, cette pratique ne doit pas être considérée comme relevant du naturel ou de l'instinct de groupe. Si elle se situe hors rituel au sens d'une pratique définie, intégrée et systématique, elle s'inscrit dans un imaginaire collectif commun de la protestation. De plus, cette gestuelle ne peut être détachée de son contexte de réalisation, porteur d'une certaine culture républicaine investissant des symboles (le drapeau tricolore), des chants (La Marseillaise) et d'autres gestuelles comme le $\mathrm{V}$ de la victoire.

\section{Arborerou brandir?}

\section{Frédéric Cépède définit le geste politique comme}

un moment suspendu, un mouvement interrompu, reconnu pour lui-même, préparé, ou qui fait irruption par inadvertance (hasard?), séparé de ce qui le précède, et de qui lui succède. Peu importe la durée, c'est une fraction de temps qui se distingue du mouvement continu, qui s'isole et se charge de sens. (2006, p.194)

Mouvement conscient et acte décomposé, le geste politique crée une rupture dans le quotidien. C'est un geste inhabituel qui s'inscrit dans un mode de communication particulier. Cependant, même codifié, il varie plus ou moins puisque chaque manifestant se l'approprie de manière personnelle avec son propre corps (ibid.). On constate tout de même une forme de permanence dans les pratiques lors des rassemblements post-attentats : le crayon est tenu entre le pouce et l'index, le reste des doigts refermés vers la paume de la main. Le bras quant à lui est levé, le coude plus ou moins plié, le niveau de fatigue jouant également dans la posture. Le crayon est pointé vers le ciel, puissant et triomphant. Ce n'est pas un mouvement bref. La pose, sans que l'on puisse déterminer de durée moyenne, est maintenue sur un temps long (de quelques minutes à plusieurs heures). Le geste s'accompagne parfois d'un mouvement de bras marquant le rythme des slogans scandés par la foule. Ce geste fait écho à une stratégie de captation de l'attention et de prise de parole : le doigt levé. À l'école, les élèves doivent pointer le doigt vers le haut pour s'adresser au professeur ou à la classe. Il rappelle également le processus du vote (à main levée). Au même titre que le drapeau tricolore, le crayon est arboré avec fierté par certains participants. En outre, on observe dans les rues de la capitale de nombreux graffitis au pochoir de ce geste dans les semaines et mois suivant les attentats (figure 1). Accompagnés ou non du slogan «Je suis Charlie», ils sont autant de marquages de l'espace public qui inscrivent dans la durée cette représentation des mobilisations pour la liberté d'expression.

On relève cependant, dans certaines représentations de ce geste (pancartes des participants aux rassemblements, graffitis et dessins de presse) ${ }^{12}$, une

12. Loïc Sécheresse et Manko, pour ne citer qu'eux. 


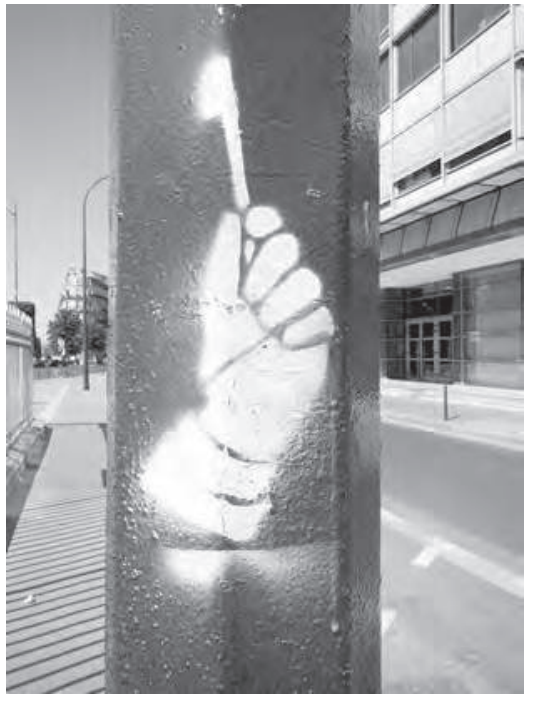

Figure 1.

Pochoir de rue, avenue de l'Observatoire, Paris, 2015. (c) Maëlle Bazin.

variante plus connotée (figures 2 et 3). Le poing replié, les doigts entourant fermement le crayon, le pouce verrouillant le tout, cette position renvoie à une tradition protestataire particulière. Brandir le poing, c'est s'inscrire volontairement ou inconsciemment dans la filiation d'un rituel politique. « Rite simple, élémentaire, qui redouble dans sa forme collective celui du mouvement spontané de menace, de colère ou de liesse, il ne nécessite aucun support «technique » et se prête à la manifestation collective comme à la pose individuelle » (Vergnon, 2005, p.78). Cette posture apparaît plus combative que lorsque le crayon est simplement tenu à la verticale. Si l'on entend par le verbe brandir le fait de «lever dans la main une arme, un objet d'un geste menaçant», on retrouve effectivement des mises en scène de cette rhétorique guerrière opposant le crayon pacifiste du dessinateur à la kalachnikov belliciste du terroriste. La gestuelle du poing dressé ou poing levé, selon les acceptions, s'est constituée en Europe en réponse au symbole fasciste du bras tendu. Le lever de poing apparaît dans les années vingt au sein de l'organisation paramilitaire du parti communiste de la République de Weimar, en Allemagne, et marque significativement le paysage politique français à partir de 1933 (Burrin, 1986, p. 11). Si le Parti communiste a été le vecteur principal de diffusion, ce geste devient rapidement le signe d'appartenance de toute la gauche: «[...] il put servir de signe de ralliement aux antifascistes de tous bords et devenir successivement l'emblème de la coalition ouvrière entre juin 1934 et mai 1935, puis celui du Front populaire tout entier» (ibid., p.12). La diffusion du poing levé est fulgurante dans les années trente-quarante avec des contextes d'utilisation relativement divers : salut, serment ou hommage funèbre. Le geste a évolué d'un «rituel 
soldatique » à un « rite de masse », notamment par son adoption en dehors des cercles militants, comme l'attestent de nombreuses photographies et actualités filmiques de l'époque. Le poing levé a traversé les décennies jusqu'à nos jours comme classique du répertoire de la contestation et de la revendication, symbole des luttes d'émancipation.

Fortement associé à la scène de l'Europe des années 1930, qui lui a donné naissance, le lever du poing s'est pérennisé à travers des contextes et des espaces fort différents, bien que toujours liés aux valeurs d'origine, des manifestants de mai 1968 et des Black Panthers américains des années 1960 aux affiches « antifascistes» des "pays de l'est» des années 1960 et 1970 ou aux récentes manifestations « anti-mondialisation ». (Vergnon, 2005, p. 78)

Ce geste a connu des réappropriations diverses au cours du $20^{e}$ siècle, diluant progressivement sa charge politique pour s'inscrire comme symbole de courage, de fierté, de liberté et de victoire à l'image du poing levé de Nelson Mandela. Le lever de poing a également été repris par les mouvements protestataires contemporains comme Occupy Wall Street et les révolutions arabes.

Régis Léger, alias Dugudus, graphiste illustrateur français, a réalisé en réaction aux attentats une affiche (figure 2) sur laquelle se détache un poing fermé sur un crayon, paume visible avec l'inscription «Nous sommes Charlie 7.01.15 "13. L'affiche, imprimée par Expressions2, a été distribuée à 20000 exemplaires aux participants des rassemblements du dimanche 11 janvier à Paris. Régis Léger évoque l’impératif de produire « un message évident, identifiable et réappropriable par tout le monde » ${ }^{14}$ compte tenu de la nature de cette affiche-pancarte. Le graphiste justifie le choix du poing et de la couleur rouge en référence à mai 1968 et aux engagements politiques de certains membres de l'équipe du journal satirique. Il ne parle cependant pas d'affiche politique mais d'affiche «historique », l'objectif étant de marquer l'événement et de créer une sorte d' «objet de souvenir» pour les manifestants. Dans cette volonté d'avoir un message clair et «sans équivoque », il fait délibérément le choix d'une représentation graphique largement usitée. Il constate à ce sujet un figement du vocabulaire visuel militant et politique sur certains codes en place depuis plusieurs années, voire décennies. Au même titre que la colombe représente la paix, le poing levé est le symbole usuel de la révolte et de la solidarité. Au regard des productions graphiques des rassemblements, il semblerait que professionnels et profanes puisent dans une sorte de catalogue imaginaire de symboles (Bazin, 2016). Cette perception dépolitisée du geste du poing levé, à laquelle s'ajoute un jeu sur les symboliques, est particulièrement parlante lorsqu'on

13. Un exemplaire de cette affiche a été récolté par le collectif des Balayeuses Archivistiques LGBT, qui a fait un dépôt d'une cinquantaine de documents au Musée des civilisations de l'Europe et de la Méditerranée (MuCEM) de Marseille.

14. Entretien téléphonique réalisé le 10 novembre 2015. 


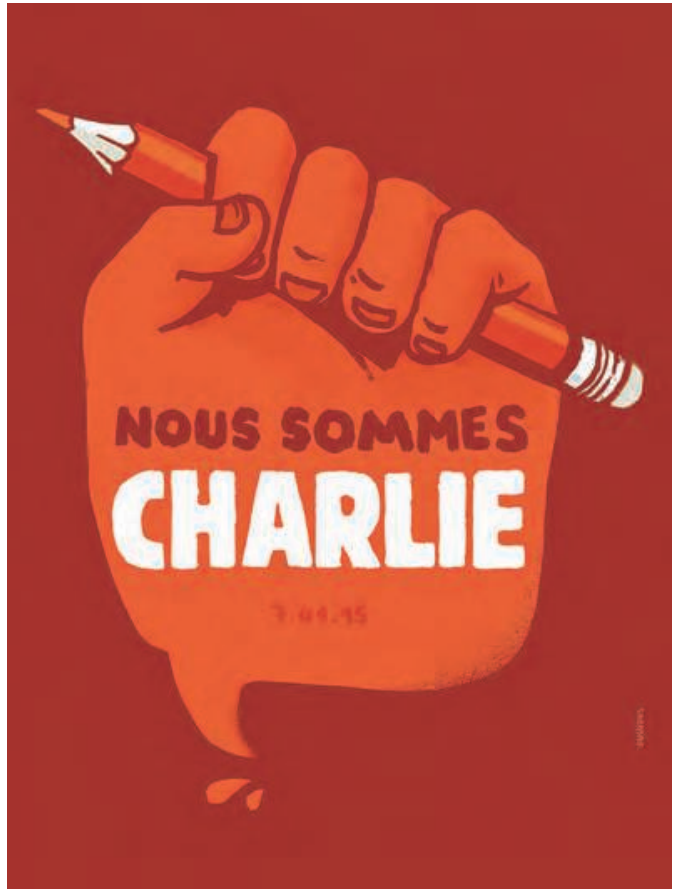

Figure 2. Affiche dessinée par Dugudus (Régis Léger) pour les rassemblements du 11 janvier 2015 (papier, $42 \times 29,5 \mathrm{~cm}$ ).

Figure 3. Pancarte, archives municipales de Saint-Étienne.

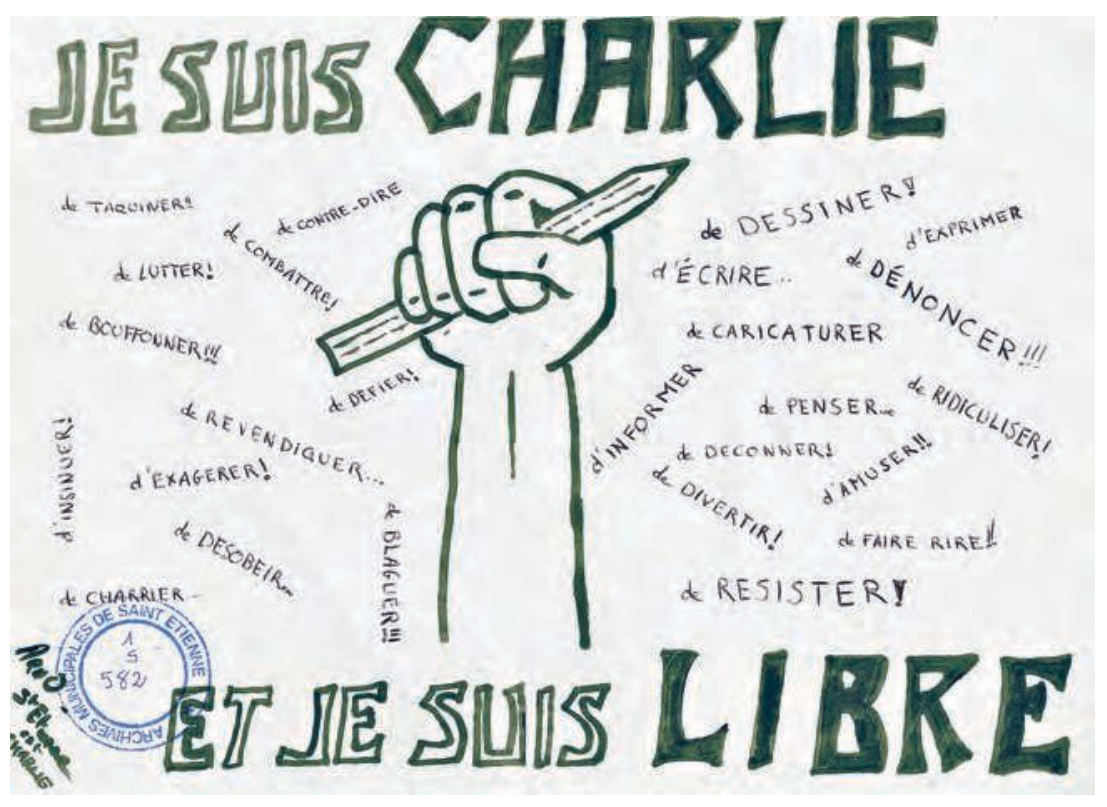




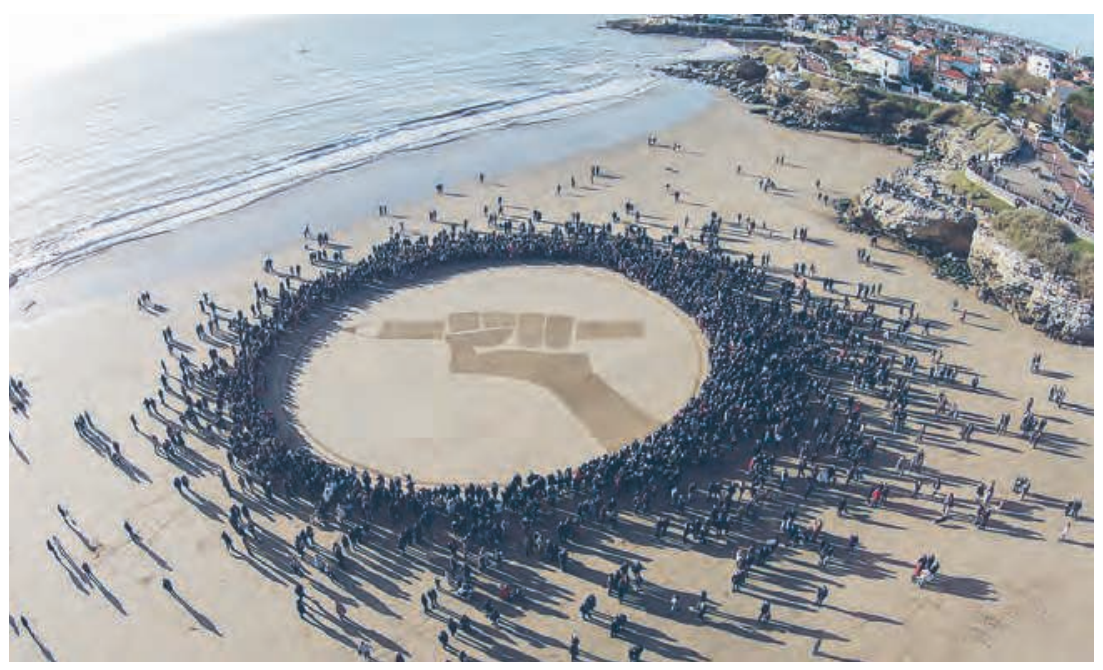

Figure 4. Dessin sur le sable réalisé par l'artiste Jben le 11 janvier 2015 sur une plage de la côte Atlantique (www.jbenart.com).

observe la performance menée par Jben, artiste français ayant réalisé un land art d'un poing brandissant un crayon sur une plage de la côte Atlantique (figure 4 infra). Il affirme s'être «inspiré d'une image trouvée sur Google»15. En hommage aux victimes des attentats, il a invité les habitants de la région à venir se rassembler autour de son œuvre ${ }^{16}$. Avant l'événement, il fournit une description de son projet et adresse des directives au public sur sa page Facebook:

Le dessin sera contenu dans un cercle. Le cercle marquera l'unité du rassemblement. Nous pourrons notamment faire un cercle humain autour du dessin. Pourquoi pas se tenir la main pour une image plus forte encore. Je vous invite aussi à emmener un crayon pour le symbole et pour ceux qui le veulent une affiche « Je suis Charlie ». L'idée est de brandir ces symboles pour la photo. ${ }^{17}$

En identifiant, verbalisant et incitant le public à brandir un crayon, il contribue à fixer cette gestuelle comme emblème des marches. On observe cependant un écart entre la représentation du poing serré et les pratiques effectives des participants qui tiennent du bout des doigts leurs crayons.

15. Entretien téléphonique réalisé le 28 mai 2015.

16. Plus de 3000 personnes sont venues pour l'événement, un chiffre bien au-dessus des espérances de l'artiste.

17. Événement : «Beach art et mobilisation pour la liberté d'expression », page Facebook de Jben beach art. Vidéo de la minute de silence : https://www.youtube.com/watch?v=7n6MwNsoX6o. 


\section{Exportation du geste}

À l'image de certaines gestuelles protestataires récentes (le salut à trois doigts des opposants thaïlandais inspiré de la trilogie Hunger Games, ou des mains levées des manifestants à Ferguson, pour ne citer que ces deux exemples récents), la gestuelle du crayon brandi caractérise une mobilisation particulière, repérable dans le temps et géographiquement. La gestuelle condense en partie l'événement ${ }^{18}$. Et à l'instar des rassemblements de sympathie à New York, reprenant le parapluie comme attribut symbolique des opposants au gouvernement chinois, on observe des gestuelles du crayon levé en dehors du territoire national dans les dizaines de mobilisations organisées à l'étranger (Berlin, Bruxelles, Madrid, Londres, Vienne, San Francisco, etc.)19. Cependant, la valeur et l'impact du crayon brandi diffèrent selon qu'il est adopté par une personne seule ou un collectif, un anonyme ou une personnalité politique, dans le cadre strict des rassemblements ou dans d'autres contextes ${ }^{20}$. Lors du discours sur l'état de l'Union de Barack Obama, le 20 janvier 2015, une quarantaine de membres du Congrès américain ont levé des crayons jaunes en hommage aux victimes des attentats ${ }^{21}$. Menée à l'initiative de Gwen Moore, cette opération illustre le passage d'une expression citoyenne à une action de communication politique. Dans un article intitulé "L'affirmation du geste dans le langage politique au $20^{e}$ siècle», Fabrice D'Almeida retrace l'évolution de la société politique sur quatre périodes passant « d'une conception du langage fortement marquée par le sens textuel à une autre, plus récente, qui voit le geste comme une production de sens déterminante et le comportement comme la clé fondamentale d'expression d'une position politique» (2006, p.156). Il met un exergue l'importance croissante du corps dans la communication politique dans nos sociétés modernes. En brandissant ainsi un crayon lors de l'allocution présidentielle, Gwen Moore affiche son soutien aux Français et marque sa position dans l'échiquier politique. Sans avoir recours au discours verbal, la politicienne adresse un signal fort devant une audience de près de trente-trois millions de spectateurs. Les organisateurs, conscients de la portée médiatique de l'action, ont travaillé la mise en scène, évacuant dans le même temps toute forme de spontanéité. Cette initiative doit être également replacée dans le contexte des controverses sur l'absence du gouvernement américain dans les rangs de la marche du dimanche, à laquelle parti-

18. Si le crayon fait clairement référence à l'attentat de Charlie Hebdo, celui-ci ne représente a priori pas les autres victimes, notamment celles de l'Hyper Cacher.

19. " "Charlie Hebdo" : plus de 100000 personnes rassemblées mercredi soir en France », 7 janvier 2015, liberation.fr.

20. Si certains maires ou personnalités politiques locales ont eu recours à ce geste, il n'a pas été repris par les membres du gouvernement français lors de la marche républicaine ou des diverses allocutions.

21. L'ovation s'est faite au moment où le président des États-Unis a fait allusion aux attentats de Paris. 
cipaient de nombreuses personnalités étrangères. À une expression collective de défense de la liberté d'expression s'ajoute ainsi une démarche que l'on pourrait qualifier de diplomatique.

\section{Le crayon guidant le peuple}

Paris, place de la Nation, des participants à la marche se massent sur le monument de Jules Dalou. Un jeune homme brandit un crayon, le drapeau tricolore se déploie au vent. Stéphane Mahé, photographe pour l'agence Reuters, immortalise la scène en contre-plongée. En quelques heures, quelques jours, l'image fait le tour du monde. Les internautes la rebaptisent Le crayon guidant le peuple (référence, bien sûr, au tableau d'Eugène Delacroix) ${ }^{22}$. On retrouve dans cette composition tous les ingrédients symboliques de cette journée : les référents républicains (le drapeau français, la statue Le Triomphe de la République), l'emblématique crayon, les affichettes «Je suis Charlie» et les manifestants nombreux, entassés. Perché sur le monument, l'homme au crayon domine la foule qui, on le sait, envahissait la place et les rues environnantes.

Dans un ouvrage publié en 2009, Clément Chéroux mobilise un terme médical, la diplopie, trouble fonctionnel de la vision qui se traduit par la perception de deux images pour un seul objet, pour définir la réitération photographique dans le traitement médiatique du 11 septembre 2001. L'auteur relève deux formes de répétition, la mise en boucle et la dimension historique de déjà-vu, qui entrent en résonance avec celles identifiées par André Gunthert pour Le crayon guidant le peuple, à savoir récurrence et référence (2015).

Récurrence, d'abord, car une deuxième image du photographe indépendant Martin Argyroglo a été renommée sous un titre identique (photographie qui a d'ailleurs fait la Une de L'Obs). Même lieu, même scène, même manifestant au crayon fidèle au poste, seule la luminosité change, le cliché étant réalisé plus tardivement dans la soirée. Deux images pour un seul objet ${ }^{23}$. Récurrence encore, car la photographie de Stéphane Mahé a fait le buzz sur les réseaux sociaux dès le dimanche soir et elle a été publiée en première page de nombreux médias internationaux le lendemain (The Times, El Periodico, La Stampa, La Repubblica, La Libre Belgique, The Daily Telegraph, et d'autres) 24.

Itération, donc, mais aussi référence. Les deux clichés ont été comparés au tableau de Delacroix et, dans certains cas, au Radeau de la Méduse de Théo-

22. Le journal Le Monde a publié le 10 janvier 2015 un dessin de Plantu détournant également La Liberté guidant le peuple de Delacroix en remplaçant les armes par des crayons.

23. D’autres images sensiblement identiques ont circulé, mais ce sont celles de Stéphane Mahé et Martin Argyroglo qui ont été les plus médiatisées.

24. Si la presse quotidienne française nationale et régionale a fait le choix de mettre en Unes des images de foule au lendemain de la marche (à l'exception du journal Le Monde) pour insister sur cette participation historique, on peut comprendre que les médias internationaux aient privilégié des photographies cristallisant davantage les symboles du 11 janvier. La présence du drapeau français permettant notamment de situer géographiquement l'événement. 
dore Géricault25, sans intention avouée de la part des photographes. Deux référents picturaux qui font partie de notre imagerie collective alimentée par les manuels d'histoire ainsi que les innombrables reprises, détournements publicitaires et artistiques. Un tissu référentiel commun aux internautes français qui parlent alors de «Delacroix revisité » ou de «version moderne de ». Le cliché de Stéphane Mahé a également été exposé sur la façade du Centre Pompidou'26. L'apposition de la parole institutionnelle d'un musée national d'art moderne légitime alors le référent artistique. "Cette image, très picturale, qui rappelle quelques icônes républicaines de l'histoire de l'art, m'a paru vivante, vibrante ", déclare Alain Seban, président du Centre ${ }^{27}$. "Elle dépasse tous les messages et illustre l'élan magnifique dont nous avons été à la fois les acteurs et les témoins ». Cependant, si l'on compare attentivement la photographie et le tableau, les éléments communs tangibles se limitent au drapeau déployé, au geste de brandir un objet, à l'attroupement et au cadrage. La liberté est un homme, la foule n'est pas armée, le contexte et l'époque sont radicalement différents. "La Liberté guidant le peuple fonctionne alors comme une matrice ou un prototype (alors que le tableau dépeint un épisode des Trois Glorieuses de 1830, il est souvent pris pour une représentation de la grande Révolution de 1789), qui fait hériter l'image destinataire de son pouvoir évocateur, à la manière d'une relique» (Gunthert, 2015). Au-delà des quelques similitudes dans la composition, c'est cette valeur événementielle qui permet le rapprochement. Le crayon guidant le peuple s'inscrit alors dans une généalogie de l'imagerie des mobilisations républicaines avec pour référent originel l'œuvre de Delacroix, nous plongeant ainsi dans une forme de paramnésie collective.

Ainsi le commentaire médiatique repose-t-il sur des indices déclencheurs de souvenirs, souvenirs de dires, de savoirs, d'événements et de représentations tels qu'ils sont reconstruits dans la mémoire de ceux qui le produisent et de ceux qui y sont exposés. (Moirand, 2007)

Dans un article au titre révélateur («Manifestations pour Charlie Hebdo: à chaque moment historique son Delacroix?"28), une journaliste de Slate.fr donne quelques exemples de mises en scènes similaires. Des élections présidentielles aux manifestations étudiantes, la statue de Jules Dalou a été maintes fois immortalisée par les journalistes pour prendre sur le vif les protestataires

25. On trouve des similitudes dans la composition, l'agglutinement de corps sur un espace réduit et dans la faible luminosité. "La statue comme mât, le gros crayon comme proue : on croirait bien voir, en effet, un bateau voguer sur la place de la Nation»: C. Bonnet, " "Charlie Hebdo" : "Le crayon guidant le peuple”, décryptage d'une photo culte», 13 janvier 2015, tempsreel.nouvelobs.com.

26. En hommage aux marches républicaines du 11 janvier 2015 , une bâche de treize mètres sur huit a été déployée le 17 janvier sur la façade du Centre Pompidou, et ce pendant plusieurs semaines.

27. «11 janvier 2015 : unis. Le Centre Pompidou rend hommage à la mobilisation nationale du 11 janvier 2015 ", communiqué de presse, 16 janvier 2015.

28. F. Arlandis, 13 janvier 2015 , slate.fr. 
en action (la place de la Nation étant un point de ralliement stratégique et historique des mouvements contestataires parisiens). Si certains lieux sont omniprésents dans les couvertures médiatiques, on relève aussi une permanence dans les gestes des manifestants.

On ne peut que s'interroger sur l'effet de la multiplication, dans la presse, d'images de groupes ou de foules levant le poing (ou tendant le bras...) dans la contagion des symboles. Pour le reporter en tout cas, comme pour le journaliste qui commente ses images, le choix de telles prises de vues marque bien la volonté de privilégier ces scènes, qu'il contribue nolens volens à rendre emblématiques. Pour le lecteur, de telles images peuvent fonctionner comme des invitations mimétiques à la pose : lever le poing face à l'objectif braqué du Leica ouvre la possibilité de se retrouver sur une photographie publiée ou dans le film d'actualités... (Vergnon, 2005)

Les choix éditoriaux sont alors moins orientés vers la valeur informative de l'image que par sa capacité à condenser un événement. La reconnaissance iconique des deux photographies Le crayon guidant le peuple et l'apposition d'un nouveau titre par les internautes fixent visuellement et verbalement le geste du crayon brandi comme emblème (Calabrese-Steimberg, 2003). On constate aussi une tentative de mythification dans le traitement journalistique avec une héroïsation de la figure de Charles Bousquet, l'homme au crayon. Reçu à la radio, interviewé par la presse, invité pour le déploiement de la bâche au Centre Pompidou, il est portraituré par les journalistes comme un jeune homme engagé, combatif et surtout courageux - resté en poste sur la statue pendant plusieurs heures. Il incarne l' " esprit du 11 janvier » et le manifestant « modèle » des marches républicaines. Certains commentateurs ont même tenté un rapprochement hasardeux entre son prénom Charles et Charlie. Cependant la mythification échoue, faute d' "exemplarité sublimée» (Boyer, 2008), dans le sens où la personnalité de l'individu ne s'impose pas sur l'événement. Elle est d'ailleurs contestée par le manifestant lui-même : «Moi je suis peut-être un symbole, mais pas un héros ${ }^{29}$. En outre, le processus de figement représentationnel connaît son apogée lorsque les internautes prédisent l'inscription dans les manuels scolaires de la photographie Le crayon guidant le peuple. À l'instar d'une membre de Twitter dont le message, comprenant l'image de Martin Argyroglo accompagnée du commentaire : "Cette photo, je la vois bien dans les livres d'histoire », a été partagé plus de huit mille fois. On trouve également d'autres énoncés similaires : «Ne cherchez plus, on a la photo qui sera dans tous les livres d'Histoire-Géo l'année prochaine», "Cette photo a de grandes chances d’être la couverture du prochain livre d’histoire de nos élèves. \#MarcheRepublicaine ${ }^{30}$.

29. C. Bouanchaud, «"Le crayon qui guide la liberté” : les coulisses d’une photo historique », 13 janvier 2015, europe1.fr.

30. M. Dehlinger, «L'histoire du “crayon guidant le peuple”, la photo symbole de la marche républicaine », 12 janvier 2015, francetvinfo.fr. 
Les deux éléments emblématiques des rassemblements post-attentats, jugés historiques par le nombre de participants, sont le slogan «Je suis Charlie » et le crayon brandi. À propos du slogan, André Gunthert parle de «polysémie proportionnelle à sa diffusion» (2015), avec une phase de consensus et une diffusion extrêmement rapide suivie de nombreuses controverses, de mouvements anti et de récupérations politiques diverses. Quant à la gestuelle du crayon, elle semble a priori beaucoup plus consensuelle, stable et linéaire dans le processus d'appropriation mais aussi plus située et limitée dans le temps et dans l'espace. Introduisant l'ouvrage Des Gestes en histoire, les auteurs soulignent l'investissement tardif des historiens dans la recherche sur le geste et un élargissement progressif du champ d'investigation à partir des années quatrevingt-dix (2006, p. 9). Dans une démarche de documentation, plusieurs archives municipales françaises (Rennes, Toulouse et Saint-Étienne) ${ }^{11}$ ont récupéré, restauré et classé plusieurs centaines de messages, pancartes mais aussi crayons manufacturés et bricolés. Ces «crayons de la paix» constituent les traces d'un événement et racontent à leur manière ces mobilisations et hommages.

\section{Références}

D’AlmeidA Fabrice, 2006, "L'affirmation du geste dans le langage politique au xx siècle», Des Gestes en histoire. Formes et significations des gestualités médicale, guerrière et politique, Paris, Seli Arslan, p.156-167.

Ambroise-Rendu Anne-Claude, D’Almeida Fabrice, Edelman Nicole éd., 2006, Des Gestes en histoire. Formes et significations des gestualités médicale, guerrière et politique, Paris, Seli Arslan.

BAzın Maëlle, à paraître (2016), «L'énonciation d'un deuil national. Usages de "Je suis Charlie" dans les écritures urbaines", Le Défi Charlie. Les médias à l'épreuve des attentats, P. Lefébure, C. Sécail éd., Paris, Lemieux (Mundo Medias), p. 153-186.

BOYER Henri, 2008, «Stéréotype, emblème, mythe. Sémiotisation médiatique et figement représentationnel», Mots. Les langages du politique, n88, p. 99-113.

BURRIn Philippe, 1986, «Poings levés et bras tendus. La contagion des symboles au temps du Front populaire », Vingtième siècle. Revue d'histoire, nº11, p. 5-20.

Calabrese Steimberg Laura, 2003, "La nomination des événements dans le discours d'information. Entre déférence et activité collective», Dire l'événement. Langage, mémoire, société, D. Londei, S. Moirand, S. Reboul-Touré, L. Reggiani éd., Paris, Presses de la Sorbonne Nouvelle, p. 233-243.

Candau Joël, Gaucher Charles, Halloy Arnaud, 2012, "Présentation. Gestes et cultures, un état des lieux», Anthropologie et Sociétés, n³ 3, p. 9-26.

CARADEC François, 2005, Dictionnaire des gestes. Attitudes et mouvements expressifs en usage dans le monde entier, Paris, Fayard.

31. Voir Archivsites!, rubrique «Carnet de bord», n¹13, 2015, p. 16-21. 
CÉPÈDE Frédéric, 2006, «Les gestes de victoire des dirigeants partisans. L'exemple des socialistes », Des Gestes en histoire, ouvr. cité supra, p. 194-208.

CHÉroux Clément, 2009, Diplopie. L'image photographique à l'ère des médias globalisés. Essai sur le 11 septembre 2001, Cherbourg-Octeville, Le Point du Jour.

CHRISTIAN Marie, 2015, Le streetartet Charlie. La mémoire des murs, Montreuil, Omniscience.

Doss Erika, 2008, The Emotional Life of Contemporary Public Memorials. Towards a Theory of Temporary Memorials, Amsterdam, Amsterdam University Press.

Fillieule Olivier, Tartakowsky Danielle, 2013, La manifestation, Paris, Presses de Sciences Po (Contester).

GINDRON Gavin, Flood Catherine, 2014, Disobedient Objects, Londres, V\&A Publishing.

GUNTHERT André, 19 janvier 2015, «Les images “iconiques” du 11 janvier, un monument involontaire?», imagesociale.fr, http://imagesociale.fr/963.

— 22 janvier 2015, "Je suis Charlie” ou les pouvoirs de l'image de soi », http://imagesociale.fr/1023.

LAMBERT Frédéric, 2007, "Les avatars de la liberté. Ouvertures sémiologiques », Analyser la communication II, A. Semprini éd., Paris, L'Harmattan, p. 69-88.

MemmI Dominique, 1998, "Le corps protestataire aujourd'hui : une économie de la menace et de la présence», Sociétés contemporaines, n³1, p. 87-106.

MoIRAND Sophie, 2007, «Discours, mémoires et contextes. À propos du fonctionnement de l'allusion dans la presse », Corela, Numéro spécial, HS-6, Cognition, discours, contextes, 2007.

Pigenet Michel, TARTAKowsky Danielle, 2003, "Les marches en France aux XIX et $\mathrm{xx}$ siècles. Récurrence et métamorphose d'une démonstration collective», Le Mouvement social, n² 202, p. 69-94.

SAnchez-CARRetero Cristina éd., 2011, El archivo del duelo, Madrid, Consejo Superior de Investigaciones Cientificas.

SOUTRENON Emmanuel, 1998, «Le corps manifestant. La manifestation entre expression et représentation », Sociétés contemporaines, n³1, p. 37-58.

StibBe Isabelle, 2015, "Ni Dieu ni maître», Nous sommes Charlie. 60 écrivains unis pour la liberté d'expression, Paris, Le Livre de poche.

Tilly Charles, "Les origines du répertoire de l'action contemporaine en France et en Grande-Bretagne », Vingtième siècle. Revue d'histoire, vol. IV, nº 1, 1984, p. 89-108.

VERGNON Gilles, 2005, "Le "poing levé”, du rite soldatique au rite de masse », Le Mouvement social, $\mathrm{n}^{\circ} 212, \mathrm{p} .77-91$.

$82 \cdot$ Le geste, emblème politique 